\title{
Philosophiques
}

Donald Martel. L'anthropologie d'Althusser. Éditions de l'Université d'Ottawa, collection Philosophica (vol. 27), Ottawa, 1984, 205 pages.

\section{Michel Dufour}

Volume 12, numéro 2, automne 1985

URI : https://id.erudit.org/iderudit/203299ar

DOI : https://doi.org/10.7202/203299ar

Aller au sommaire du numéro

Éditeur(s)

Société de philosophie du Québec

ISSN

0316-2923 (imprimé)

1492-1391 (numérique)

Découvrir la revue

Citer ce compte rendu

Dufour, M. (1985). Compte rendu de [Donald Martel. L'anthropologie

d'Althusser. Éditions de l'Université d'Ottawa, collection Philosophica (vol. 27),

Ottawa, 1984, 205 pages.] Philosophiques, 12(2), 459-462.

https://doi.org/10.7202/203299ar d'utilisation que vous pouvez consulter en ligne.

https://apropos.erudit.org/fr/usagers/politique-dutilisation/ 
DONALD MARTEL. L'antbropologie d'Altbusser. Éditions de l'Université d'Ottawa, collection Philosophica (vol. 27), Ottawa, 1984, 205 pages.

par Michel Dufour

Plusieurs éprouvent une certaine inquiétude devant les livres-thèses. On y redoute le ton académique, l'abus des citations, la facture traditionnelle, le style poli, la neutralité plus ou moins scientifique, etc. Or, la collection Philosopbica ne se distingue pas par des couvertures racoleuses. Le sérieux qu'elles promettent habituellement se confirme dans ce cas-ci, dès la table des matières : il y est question d'Althusser et de Lacan, du mode paranoïaque de production de connaissance, d'un discours sans sujet et décentré, d'une seconde conscience ...

En un sens, ce livre sent un peu la "thèse ", mais le titre aurait déjà dû nous apprendre à nous méfier de notre méfiance. Par ailleurs, il est vrai que l'auteur manifeste une sobriété passionnée, au sens où il s'en tient à son sujet (si on peut dire) et nous épargne coquetterie de style, maux de cœur et prophéties apocalyptiques ou lénifiantes. Bref, on finit par se laisser prendre, on s'aperçoit qu'il s'agit là d'un livre que plusieurs ont voulu écrire depuis une dizaine d'années, ne serait-ce que pour mettre un peu d'ordre dans cette vague structuraliste qui a déferlé sur le milieu intellectuel québécois des années 70 . Avec une clarté et une précision remarquables, l'auteur recompose une problématique fondamentale pour la compréhension de la philosophie française contemporaine (constitutive, entre autres, de la thématique de la mort de l'homme). Les qualités pédagogiques ne suffiraient pas à expliquer qu'on lise ce livre un peu comme un roman policier. Un peu. Chaque chapitre apparaît comme la pièce d'un puzzle ; chaque pièce est habilement découpée et les enchaînements soignés ménagent le suspense.

Voyons l'itinéraire. Le titre indique le fil directeur, mais c'est en même temps un clin d'œil, sorte d'" inside joke " : l'anthropologie (!) d'Althusser. L'auteur va tellement se confondre à Althusser par la suite qu'il nous avertit que, même si son travail présuppose une disponibilité totale à Althusser, il n'en garde pas moins personnellement (si on peut dire) une certaine distance critique : Althusser a passé une partie de son temps à dénoncer la possibilité même d'une anthropologie, alors que l'auteur tente de dériver de la théorie althussérienne, d'y lire symptômalement, une sorte d'anthropologie non anthropocentrique. C'est peut-être en réduisant un peu le sens 
d'« anthropologie » que l'auteur y parvient, mais ce n'est certes pas en trahissant Althusser.

Le premier chapitre (Anthropologie et anti-humanisme chez Althusser) rappelle quelques acquis et développe le rapport entre l'humanisme et la question du sujet. L'intervention althussérienne, au moins dans ses effets et probablement dans ses causes, s'inscrit dans le problème du rapport entre les militants intellectuels et les militants ouvriers ou paysans. Comment tailler une place à l'intérieur du mouvement prolétarien et du Parti communiste français pour des intellectuels petits-bourgeois marxisants et de bonne volonté, qui parviennent plus ou bien à se contenter de servir de relais de transmission à des dogmes, ou bien à renoncer à leur spécificité de penseur (rapport à la vérité) au profit d'une explicitation systématique et d'une diffusion massive des idéologies progressistes (rapport à l'efficacité) ? Sans nier la ligne de démarcation entre idéologies progressistes et idéologies réactionnaires, Althusser dessine l'opposition, plus fondamentale au niveau théorique, entre Idéologie et Science. Une idéologie n'est pas simplement une conception du monde, c'est une représentation tronquée à la base, une fausse conscience, tandis que la science se constitue et se définit comme critique de la conscience idéologique et mise en place d'une problématique où les réponses cesseront d'apparaître comme des pétitions de principe (reflet spéculaire). Or, ce qui caractérise l'Idéologie en général, c'est une méconnaissance originelle et éternelle dont le noyau conceptuel s'apparente à l'Humanisme, c'est-à-dire à une façon de penser la liberté ou la volonté comme le centre de l'Homme, et l'Homme comme sujet de l'Histoire et propriétaire de ses émotions et de son discours. D'où la nécessité d'un retour à Marx, mais au Marx scientifique qui rend possible la science de l'histoire, plutôt qu'au jeune Marx encore empêtré dans l'humanisme, critique mais anthropocentrique, de Feuerbach. L'anthropologie, pseudo-science humaine, est donc mal partie, puisqu'elle a tenté jusqu'ici ou bien de définir l'être humain en termes de substance et d'attributs, ou bien de saisir l'essence générique de l'être humain à partir de ses productions considérées comme expression de la personnalité du sujet ou de l'originalité d'un sujet collectif.

Cependant, et ici vient le chapitre sur Lacan (L'Imaginaire et la conscience paranoïaque), comment se réalise cette méconnaissance originelle ? Lacan, dont l'effort consiste à prendre l'inconscient à la lettre, a montré l'importance du stade du miroir dans la formation du Je de l'enfant, qui accède ainsi à l'Ordre du Symbolique, ou du Culturel dirait Althusser, qui dit aussi toutefois que l'Idéologie est éternelle, omnitemporelle, bref irréductible aux aléas de la lutte des classes. L'Idéologie fonctionne quand l'être humain se prend pour un sujet ou un centre, et la psychanalyse explique comment le passage à l'Humanité s'effectue à partir du moment où l'enfant se prend pour un autre, se " reconnaît " dans l'image spéculaire, se saisit comme sujet (Je), autour duquel le monde s'organise et à partir duquel le sens vient au monde. L'image et l'idéologie apparaissent comme des lieux de méconnaissance produisant des effets de connaissance idéologiques. Téléscopant l'image et l'idéologie, l'individu et la société, l'auteur rapproche 
donc matérialisme historique et paychanalyse, dont les analogies sont d'ailleurs relevées par Althusser lui-même.

Les deux chapitres suivants explicitent l'idée d' « effet de connaissance idéologique " en suivant à la loupe les travaux d'Althusser : l'auteur reconstruit minutieusement la stratégie de la coupure qui conduit à distinguer le Marx des Manuscrits de 44 du Marx du Capital (1867), davantage en possession de ses moyens, en passant par l'ambigu règlement de comptes de L'Idéologie allemande (1845). L'essentiel du changement de terrain consiste justement à produire un nouveau type de discours, un discours sans sujet et décentré, où les rapports sociaux de production sont impensables en termes de sujet(s).

Or, n'y a-t-il pas moyen, ces acquis assumés, de constituer une anthropologie non anthropocentrique, et Althusser n'a-t-il pas justement besoin d'une telle anthropologie pour fonder, ne serait-ce que méthodologiquement, sa théorie des idéologies? En tant qu'historien, on peut se contenter de prendre acte du fait que l'idéologie est toujours déjà là et qu'elle est éternelle ; mais en tant que méta-théoricien, il faut articuler l'articulable. On pourrait ainsi finir par comprendre, par exemple, que pour que la philosophie et le droit bourgeois réussissent à populariser une conception du monde où des sujets libres s'associent librement pour imprimer leur être profond dans l'État, la Culture et l'Être, il faut que cette vision réactive quelques-uns de nos phantasmes. Il faut que la « connaissance " idéologique vienne relayer la méconnaissance première définie par la psychanalyse. Et, s'il est vrai que même la psychanalyse lacanienne comprend une certaine forme d'anthropologie, au sens où si l'être humain doit être pensé comme passivité comme champ de forces, il faut bien que quelque chose soit pensable, alors il semble qu'Althusser doive nuancer sa critique de toute anthropologie et assumer plutôt l'anthropologie plus ou moins explicite de Lacan.

Dans un tel contexte, comment se définira la pratique philosophique? C'est, suite à un moment de défaillance intellectualiste où elle avait paru être Théorie de la pratique théorique, la lutte des classes ou la politique dans la théorie, c'est-à-dire la lutte impitoyable menée contre toute résurgence de l'humanisme théorique. Ce qui conduit à la question capitale : " comment sortir de cette conscience idéologique ? ". L'auteur comprend cette question au niveau stratégique : contre le spontanéisme de Lukacs, Lénine est convoqué. S'imaginer que les masses, par une pirouette dialectique, transformeront par elles-mêmes leur méconnaissance en conscience juste, c'est succomber à la forme idéaliste du mécanisme. L'intellectuel marxiste est nécessaire : pas de mouvement révolutionnaire sans théorie révolutionnaire, mais aussi pas de théorie révolutionnaire sans théorie scientifique.

Dans l'ensemble, la démarche est accomplie avec une grande maîtrise. La thèse elle-même me semble aller de soi. La mise en place de la grille conceptuelle althussérienne s'avère très précieuse et permet même de comprendre mieux la position dite «structuraliste » commune à Lévi-Strauss, Lacan et Foucault. On me permettra peut-être une ligne de fuite en guise de pseudo-conclusion. On aurait pu comprendre autrement la question : 
comment sortir de cette conscience idéologique ? Comment l'intellectuel scientifique marxiste a-t-il pu lui-même se constituer comme tel ? Doit-on faire à Althusser le reproche jadis adressé à Hegel, à savoir que sa théorie peut rendre compte de tout, sauf d'elle-même ? Comment expliquer qu'on est le seul à avoir le pas dans la grande marche ? En d'autres mots, si le passage entre la première conscience (la conscience imaginaire et la méconnaissance typique qui lui est corrélative) et la seconde conscience (la conscience juste et la connaissance scientifique qui lui est liée) ne s'accomplit pas par mutation brusque, une anthropologie, anti-humaniste tant qu'on voudra, ne devrait-elle pas pour être complète pouvoir décrire aussi bien les conditions d'émergence de la conscience juste que celles qui permettent de comprendre la transformation d'une conscience diffuse (de) soi en conscience inconsciente ? Sinon, le bébé qu'on jette avec l'eau du bain, c'est la dialectique.

Département de philosophie

Collège Maisonneuve 